\title{
LA FENOMENOLOGÍA DE LA AFECTIVIDAD DE DIETRICH VON HILDEBRAND: UNA PROVOCACIÓN CORDIAL PARA LA TEOLOGÍA
}

\author{
DIETRICH VON HILDEBRAND'S PHENOMENOLOGY OF \\ AFFECTIVITY: A HEARTED PROVOCATION FOR THEOLOGY
}

\author{
Dr. Ricardo Mejía Fernández ${ }^{1}$ \\ Universidad de Salamanca, España \\ University of Memphis, USA
}

\begin{abstract}
Resumen: En el presente estudio pretendo recuperar la fenomenología de un pensador todavía por descubrir en nuestro ámbito iberoamericano. Dietrich von Hildebrand (18891977), nacido en el mismo año que Martin Heidegger y tras haber sido pupilo de Max Scheller y Edmund Husserl, trabajó con brillantez en el método fenomenológico. No explicaremos aquí la fenomenología fundamental hildebrandiana, sino su fenomenología aplicada a la afectividad, en cuanto que sutil provocación para la teología. Este trabajo retratará, por tanto, una fenomenología especial y no general; dedicándonos a la cualidad personal de una afectividad a la vez diversa y unitaria.
\end{abstract}

Descriptores: Fenomenología $\cdot$ Afectividad $\cdot$ Sentimientos $\cdot$ Pasiones $\cdot$ Hildebrand $\cdot$ Teología.

\begin{abstract}
In the present study, I would like to recover the phenomenology of a thinker that remains still undiscovered in the Ibero-American realm. Dietrich von Hildebrand (18891977) was born the same year as Martin Heidegger, and was a disciple of Max Scheler and Edmund Husserl, working brilliantly in his phenomenological method. I am not going to explain here the fundamental phenomenology of this author but his phenomenology as it is applied to affectivity, in order to subtly provoke theology. This paper, then, is going to take notice of a special phenomenology and not of a general one, by dedicating our efforts to the personal quality of a diverse and unified affectivity.
\end{abstract}

Keywords: Phenomenology $\cdot$ Affectivity $\cdot$ Feelings $\cdot$ Passions $\cdot$ Hildebrand $\cdot$ Theology

\section{INTRODUCCIÓN}

En el presente estudio queremos rescatar una figura inicuamente olvidada en la filosofía contemporánea, sobre todo en nuestro ámbito iberoamericano. Dietrich von Hildebrand (1889-1977) nació el mismo año que Martin Heidegger y fue también pupilo de Max Scheler y Edmund Husserl, trabajando con brillantez en su método fenomenológico. A diferencia del colaboracionismo de Heidegger, Von Hildebrand fue un implacable opositor del nazismo, si bien ha permanecido en

\footnotetext{
${ }^{1}$ E-mail: ricardomejia@usal.es
} 
la sombra quizá a causa de sus adherencias explícitamente religiosas. El católico Von Hildebrand le plantó cara a la ideología nazi con más tenacidad que muchos de sus compatriotas y colegas filósofos, hasta el punto de ser perseguido y tener que exiliarse a los Estados Unidos. En My battle against Hitler, Von Hildebrand (2014), p. 33, denunciaba, en pleno ascenso al poder de esta ideología, que su país "había caído en manos de los criminales" debido al "triunfo de este horrible ultranacionalismo y su profundo espíritu anticristiano".

En clara distancia del nacional-catolicismo pero también del nacionalismo alemán basado en la superioridad racial, el pensador defendió un "espíritu católico supranacional" (supranational catholic spirit) (Von Hildebrand, 2014, p. 25), que no estuviese atado a los ídolos sentimentales del pueblo y la raza pero no por ello siendo enemigo de la afectividad. Esto provocará también a la teología. La fenomenología de la afectividad que ahora estudiamos es una tajante contestación al desbarajuste afectivo de un discurso (sea político, filosófico, teológico...) basado en la incitación sentimentalista de la gente y no en la armonía hacia el pensamiento riguroso y los valores morales. Muchas falacias provenidas de las diversas instancias públicas y religiosas se deben a la carencia de analizar fenomenológicamente el sentido, alcance y vivencia misma de la afectividad. Por esto, aquí analizaremos el trabajo que Von Hildebrand publicó, primero en 1965 y finalmente en 1977, con el título The heart: an analysis of human and divine affectivity; dando cuenta de la actualidad que todavía tiene el filósofo alemán en este tema. Suscribimos, por ello, las palabras de Juan Manuel Burgos (2009), p. 7, el relevante personalista español y traductor del fenomenólogo alemán, cuando afirmaba que la citada obra "nos parece de particular actualidad"; precisamente ante la mezcolanza contemporánea de las pasiones y sentimientos más bajos - dignos también de exploración fenomenológica- con los estados más excelentes de la persona.

Si bien dicha obra tiene tres partes en continuidad recíproca - una filosófica y dos de temática más clásicamente teológica- aquí nos ceñimos, por la limitación de espacio de este artículo y el tipo de revista en la que publicamos, a la primera parte sobre la fenomenología de la afectividad. Tampoco explicaremos aquí la fenomenología fundamental de Von Hildebrand, que bebe de Scheler y Husserl, sino su fenomenología aplicada a la afectividad como provocación cordial para la teología. Nuestro trabajo, pues, da cuenta de una fenomenología especial y no general. De todos modos, y recordando unas palabras de Von Hildebrand, a propósito de su fundamentalidad y generalidad, leemos en What is Philosophy? que "la fenomenología es la total antítesis de la tendencia anti-cualitativa (...)" al involucrar en esta guisa "una total receptividad para la esencia de la fragancia de lo espiritual, de las entidades culturales en toda su plenitud existencial y cualitativa" (Von Hildebrand, 1973, p. 226). 
En este artículo, y en tanto que filósofos, no vamos a hablar del pensamiento teológico clásico sino de la fenomenología hildebrandiana, la cual consagró a la cualidad personal -cordial, más profundamente- de la afectividad como punzante provocación para la teología. En consecuencia, nuestro artículo pertenece y se acotará en este dominio fenomenológico y personalista, si bien sabemos que hoy se ha de apostar por la transdisciplinariedad. La brevedad nos obliga, así como el deseo de que pueda ser relevante el aspecto más fenomenológico de este pensador para un público más amplio -especialmente teológico- y tal vez no formado en temas fenomenológicos. No es, en suma, nuestro artículo un trabajo teológico sino provocativo indirecte para la teología.

\section{El CENTRO DE LA AFECTIVIDAD: EL CORAZÓN}

Von Hildebrand se lamenta de la ausencia de reflexión en torno a la afectividad en la historia del pensamiento occidental, siendo este tema el gran y más atroz ausente entre los filósofos y bastante desencarnado entre los teólogos. Además de desvelar su abandono, el alemán no cejó en su empeño de reconocer su eminente centralidad: "La esfera afectiva, y el corazón como su centro, ha estado más o menos bajo una nube a lo largo de la historia de la filosofía" (Von Hildebrand, 2009, p. 31). El corazón, en efecto, es el centro de la afectividad personal humana y debería serlo de cualquier empresa que se diga y se tenga a sí misma como humana; no siendo la filosofía un punto de inflexión.

Y es que, entre filósofos y teólogos, el corazón ha sido repulsado como "el hijastro", sufriendo una suerte de bastardía que ha repercutido muy negativamente en las ideas occidentales ya que "no se ha concedido ningún espacio a la exploración del corazón (...). La esfera afectiva, y con ella el corazón, ha sido excluida del ámbito espiritual" (Ibid.). En la tradición racionalista, desde Platón y pasando por Aristóteles, "la esfera afectiva, y con ella el corazón, pertenecen a la parte irracional del hombre" (Ibíd.); habiendo muy desafortunadamente un "lugar inferior reservado a la afectividad en la filosofía" (Von Hildebrand, 2009, p. 32). La afectividad ocuparía, así, un lugar de segundona entre las preocupaciones de los filósofos, al insistir estos de manera bien retraída "en su carácter irracional y no espiritual" (Von Hildebrand, 2009, p. 33).

Entre las causas de este desprecio se encuentra la confusión de la afectividad con sus deformaciones más bajas, algunas con un valor moral negativo. Contra esta "falsa interpretación" (Von Hildebrand, 2009, p. 34), la afectividad no solamente comprende las pasiones deleznables sino también aquellas que son implicadoras del espíritu más liberador. De hecho, "la razón más contundente para el descrédito en que ha caído toda la esfera afectiva se encuentra en la caricatura que se produce al 
separar una experiencia afectiva del objeto que la motiva y al que responde de modo significativo" (Von Hildebrand, 2009, p. 36). Hay, pues, motivaciones implicadas por la afectividad que no la desvirtúan, sino que la elevan en su auto-tendencia intrínseca. El alemán piensa que, si bien en el caso del cristianismo -como en los Padres griegos- se da cierta continuidad al racionalismo griego, al menos ya se empezaba a desbiologizar el papel del corazón (es decir, a eludir la concepción desdeñosa del mismo como pura animalidad y no apto para la dignidad humana), debido a la importancia que se le concedía al dogma cristiano de la encarnación. No es que Von Hildebrand (2009), p. 38, niegue la importancia de las ciencias empíricas sino la falsa biologización del corazón, en tanto que totalmente ajeno al ser humano racional; lo cual no sería más que "una desnaturalización" en sentido reduccionista. Nadie, en su condición humana saludablemente viviente, puede renunciar a su afectividad sino solamente reprimirla.

De acuerdo con la fenomenología hildebrandiana, hemos de evitar, en la medida de lo posible, no confundir la afectividad con sus orientaciones perversas; las cuales el alemán sintetiza en tres maneras fenomenológicas fundamentales: (1) identificar la afectividad con su objeto, lo cual es falso porque es el objeto el que depende de la potencia afectiva primera; (2) separar totalmente la afectividad del objeto, lo cual lleva a una afectividad convertida en placer de superficie tenido como un absoluto, esto es, como "falsificación de su misma naturaleza" (Von Hildebrand, 2009, p. 39); (3) la confusión de la afectividad con el "sentimiento de responsabilidad" (Ibid.), perdiendo su substancia en nombre de una moral formal y aparentemente autónoma.

Y es que, para que nos podamos sobreponer a estas perversiones, tendremos que mantener, contra viento y marea, que la afectividad "no tiene por qué tener necesariamente un nivel ontológico menor que su objeto respectivo" (Ibid.). El objeto no resta nada a la afectividad en todo su poder in se sino que, más bien y particularmente, degrada su puesta en acto. Es, entonces, la mala práctica (hacia un objeto nocivo o mediante una intensidad desproporcionada y desequilibrada) de lo degradado lo que daña a la persona.

\subsection{EL CORAZÓN COMO YO REAL}

La postura de Dietrich von Hildebrand para con la efectividad es no solamente mucho más positiva que el racionalismo teológico de Occidente sino que forma parte de su proyecto el descubrirla e integrarla en el núcleo de la persona. En la preclara argumentación fenomenológica del alemán, desabrigamos un primor de la afectividad que le hace sobresalir en comparación con sus pares; principalmente en el dominio filosófico alemán de los la década de los sesenta. La afectividad o el 
corazón no es aquel tema aledaño que podremos, algún día y ya aburridos de tratar las capacidades intelectivas o sociales según el marxismo de esta época, abordar casualmente en nuestra reflexión.

Von Hildebrand, del lado del pensamiento teológico hebreo del lev, asevera sin remilgos que "el corazón constituye el yo real de la persona más que su intelecto o su voluntad" (Von Hildebrand, 2009, p. 133). Podemos ser todavía más resueltos al afirmar que el corazón "constituye la parte más íntima de la persona, su núcleo, el yo real" (Ibid.). Nada, pues, en la persona humana se vive fenomenológicamente con más realidad y determinación que la afectividad, en discrepancia de la definición que realizó Boecio "en el tercer capítulo de su Sobre la persona y las dos naturalezas, donde expresaba: Persona est naturae rationalis individua substantia" (Culleton, 2010, p. 61), esto es, como sustancia individual de naturaleza racional. En el alemán, por el contrario, la persona se experimenta con más premura que como una res racional, existente o incomunicable: su quicio primordial es la afectividad. Así pues y siendo también denominada "esfera afectiva", en y desde la misma podemos franquear fenomenológicamente dos niveles (Von Hildebrand, 2009, 137):

1) Estados afectivos simples. Este es el nivel de los sentimientos y las pasiones en un surtido de variantes, las cuales "se colocan por debajo de los actos que pueden ser alcanzados inmediatamente por nuestra libertad" (ibid.). Aquí se descubren estados afectivos tanto corporales (cansancio, sueño, etc.) como psíquicos (buen humor, depresión, etc.). Fenomenológicamente descubrimos que estos estados tienen un grado ontológico menor que el propio de los estados superiores, como veremos en seguida.

2) Estados afectivos profundos. Aquí entroncamos con "un nivel más elevado en la esfera afectiva" (ibid.), entendiendo por "elevado" no lo que cae fuera de la naturaleza humano sino en el sentido de la interioridad pre-reflexiva, el yo subterráneo de las "respuestas afectivas que vienen de lo más profundo del alma de la persona" (Ibid.). Este nivel funda los actos volitivos pero no es paralelo a la voluntad: lo vivimos, usando otra expresión, como su propio poso. Tampoco se identifica con el subconsciente porque este no deja de ser regional con respecto a la persona entera. Como rubrica el alemán, esta afectividad más radicalmente cordial "no la poseemos (...) no cae bajo nuestro dominio" (ibid.), sino que nos abarca íntegra e intrínsecamente.

Por consiguiente, no experimentamos fenomenológicamente este nivel como un dato entre otros de nuestra interioridad sino bajo el tamiz del "carácter de un don de lo alto" (ibid.), como algo que nos es donado y es imposible de encorsetar en categorías racionales que, si hablamos en propiedad, le son derivadas. Esta experiencia fenomenológica del don, en la afectividad más fontanal del 
corazón, es una muestra, a un nivel simplemente natural, de que como humanos nos vivimos desde el don de forma misteriosa; lo cual está en estrecha analogía (aunque no identificación) con una teología natural cristiana que estudia "los dones naturales de Dios que el hombre no puede darse a sí mismo" (Von Hildebrand, 2009, p. 138). Pero no nos distraigamos: estamos en el ámbito natural al método fenomenológico y para nada directe en el de la teología. Estos dones naturales "proceden de lo más profundo" de la persona "son, de un modo particular, las voces de su verdadero yo, de la plenitud de su ser personal” (ibid.).

Habida cuenta de esto, el alemán objeta que el nivel afectivo profundo se identifique con la moralidad humana ya que más bien la segunda es deudora del primero: las acciones conscientemente libres y responsabilizadas se nos ofrecen antes, durante y después de nuestros actos como afectivas. La afectividad cordial, que a veces la vivimos en la forma de una felicidad inexplicable cuando actuamos de acuerdo con la moral, “es < más bien> una manifestación más profunda del verdadero yo de una persona que una manifestación de su libre centro espiritual" (Von Hildebrand, 2009, p. 134). Desde la publicación de Christian Ethics (1953), Von Hildebrand explicaba que la manifestación más clara de la autenticidad de la libertad humana era la "libertad cooperativa" de la "aceptación (sanctioning) en el 'sí' de nuestro libre centro espiritual", el cual pertenece al yo profundo del corazón (Von Hildebrand, 2009, p. 138). La moral teológica se adscribe naturalmente a ello, de suerte que las solas acciones que podemos sancionar como morales son las "respuestas afectivas" ante la "voz de nuestro corazón que es un don de lo alto" (Ibid.).

Lo moral, más que el vacuo y categórico imperativo kantiano, es lo que respecta a la afectividad en su autenticidad, convertido "en la palabra expresa y plena de nuestro propio yo" (Von Hildebrand, 2009, p. 139). Esto significa que la fenomenología del corazón o la afectividad es más apremiante que la moral supraindividual, de tal manera que si no hacemos una philosophia prima del nivel fontanal afectivo no sería posible hablar con una base humana auténtica de actos morales. A continuación, veremos que no siempre contamos con tal autenticidad, de suerte que nuestra manera de actuar en relación con la afectividad puede depravarse.

\section{TRES TIPOS DE TEORÍAS INAUTÉNTICAS DE LA AFECTIVIDAD}

En consecuencia, con el anterior punto de la afectividad como centro de la persona y de la calificación moral de sus acciones, hemos de estar prevenidos contra las "teorías equivocadas" de la afectividad; las cuales son causantes de tres grandes tipos de su "falta de autenticidad" (Von Hildebrand, 2009, p. 40) 
y dan lugar a formas de deshumanización que menoscaban a las personas que las sufren o las fomentan. Veamos, sucintamente, estos tipos con la ayuda de la fenomenología hildebrandiana:

1) Falso pathos retórico. El alemán describe esto como "falta de autenticidad retórica", en la que el ser humano hincha con su verbo sus sentimientos, "su falso pathos es, en sí mismo, una continua fanfarronada emotiva” (Ibid.). Se trata, a la sazón, de la ampulosidad decimonónica y romántica, aunque todavía actual, que tiende a reducir la efectividad a una narración sobrecargada que, a pesar de su copioso ornato verbal, no garantiza su autenticidad afectiva: "Al deleitarse en sus profusos e hinchados sentimientos se descentra en cuanto se enfrenta con un objeto real y con su tema", todo ello sin carecer inclusive de "un exhibicionismo característico de quien disfruta desplegando este pathos ante una audiencia" (Von Hildebrand, 2009, p. 41). Podríamos añadir a las palabras del alemán, además, que la forma y los receptores de un discurso no garantizan lo auténtico del contenido de lo declamado; ni mucho menos de su residuum afectivo. En este primer tipo de inautenticidad afectiva se da una suerte de esteticismo que busca, hasta el cansancio del deleite ajeno, exteriorizar y componer de una manera tan manifiestamente sensible, que nos hace poner seriamente en duda su veracidad y verdad en el core afectivo.

2) El solipsismo. Otro ejemplo de inautenticidad afectiva es el de "una profunda inmersión en uno mismo" (Ibid.). Nos situamos, pues, ante la antítesis del primer tipo pero no por ello ante una menor falsificación. Si bien no vemos en este caso la jactancia verborreica anterior, sí que hay un goce egoísta de las posibilidades y afecciones variadas de la afectividad. Como se puede adivinar, "el rasgo específico de esta falta de autenticidad estriba en que, en lugar de centrarse en el bien que nos afecta o que origina una respuesta afectiva, la persona se centra en su propio sentimiento" (Ibid.). La afectividad es trastocada de nuevo por el sentimiento volcado a su objeto de goce de una manera totalmente privada y solitaria. De esta manera, "lo que debería ser algo que nos afecta intencionalmente, queda así degradado a un puro estado emocional originado y activado por un objeto" (Ibid.).

En esta coyuntura, la persona no quiere encarar el significado intencional y relacional de sus sentimientos en relación con sus otras dimensiones personales, dándose "la 'pobre cualidad' de la emoción experimentada y del objeto que la provoca" (Von Hildebrand, 2009, p. 42). Se trata de la "perversión introspectiva" que generan determinadas experiencias religiosas como la que el alemán llama con gran dureza "la orgía de la 'contrición' de algunas sectas religiosas” (Ibid.), esto es, el complacerse con el dolor de los pecados como fin insano en sí mismo. Esta orgía como la llama el filósofo- se da en la falsa piedad contritiva, característica de "sentimientos pseudo-religiosos" (Von Hildebrand, 2009, p. 46) que no buscan la profundidad espiritual de una experiencia cualitativa sino la emoción cuantificable por sí 
misma. Es, en suma, un "sentimentalismo" descrito como un "disfrute introvertido" que, desafortunadamente, "causa necesariamente una perversión cualitativa" (Von Hildebrand, 2009, p. 45) de la vivencia que sancionaría una teología de este tipo.

3) El histerismo. Von Hildebrand nos aclara que esto no es directamente la patología histérica tal y como consta en los catálogos psiquiátricos en la forma de trastorno de conversión neurótico. Estamos ante un tipo de inautenticidad afectiva que, aunque no implique necesariamente déficits psicológicos o neurológicos, pertenece más bien al orden fenomenológico fundamental: "Nos referimos a aquellas personas encerradas en un egocentrismo excitable" (Ibid.). A diferencia de los dos tipos anteriores (el totalmente patético-retórico o el solipsista), en esta perversión de la afectividad comprobamos una mezcla de los dos: el exhibirse ante los demás o ante sí mismo causa un placer desordenado de tal modo que tanto "el orgullo como la concupiscencia están en la base de esta perversión" (Von Hildebrand, 2009, p. 47). Así, la persona vive para lucirse ante los otros y cambia sus estados de ánimo en función de una instrumentalización de sí misma a favor de su auto-imagen y la consecución de beneficios, a corto y a largo plazo, en el medio social.

Es, por tanto, una afectividad mal orientada al no sacar partido de sus mismas potencialidades con el fin de lograr ahondar y crecer en todas sus dimensiones humanas. De esta manera, se llega a la mentira o a la exageración, tan solo "por satisfacer su deseo peculiar e incansable de estar en primera línea, de desempeñar un papel, de hacerse las interesantes no solo para las demás, sino también para sí mismas" (Ibid.). Y es que querer llamar la atención por el goce de hacerlo "se manifiesta en una volubilidad que entremezcla verdad y mentira" (Ibid.).

Pero, ¿cuál es la forma afectiva que podemos identificar como totalmente contraria contra el histerismo afectivo? Von Hildebrand la describe como "una respuesta afectiva profunda y genuina, un amor verdaderamente luminoso o una alegría santa" (Von Hildebrand, 2009, p. 50); lo cual indica que no toda vivencia de la afectividad rebaja nuestra condición humana. Por consiguiente, no debemos confundir ni dualizar los tipos inauténticos de vivencia de la afectividad con la afectividad total y con aquellas vivencias encaminadas a aumentar nuestras cualidades humanas más excelentes. Vencer esta confusión y dualidad es lo que estudiaremos en el siguiente punto.

\section{LA OSCILACIÓN ENTRE EL CARÁCTER ESPIRITUAL} Y NO-ESPIRITUAL DE LA AFECTIVIDAD

Es constatable que, ante los anteriores tres tipos de perversión afectiva, la persona experienciante y la filosofía que emana de su experiencia haya oscilado entre, por un lado, una afectividad no-espiritual y, por otro, una afectividad espiritual. Es 
como si algunos entendiesen la afectividad solo como aquellas pasiones más viles del ser humano, mientras que para otros fuese un puro goce subjetivo. Por tanto y para sobreponerse a esta dualidad, Von Hildebrand prefiere el término "corazón" en cuanto que, ya inicialmente y de una manera semántica, denota tanto un órgano fisiológico humano como su condición espiritual.

Al decir "corazón", aplicamos significados cualitativos y cuantitativos a la afectividad humana, y esto es lo que nos importa. Lo semántico, en efecto, hace honor al método fenomenológico en este autor. "Corazón", en Von Hildebrand (2009), p. 47, designa tanto "la vida interior del hombre en cuanto tal" y "es más o menos sinónimo de alma". En el alemán, el corazón implica voluntad e inteligencia ya que hemos de tener claro que "la afectividad es una realidad importante en la vida de la persona y que no puede ser subsumida en el intelecto o la voluntad" (Von Hildebrand, 2009, p. 58). Así, el corazón expresa de manera conspicua algo más integrador que la dualidad espiritual/no-espiritual en la afectividad, siendo de suyo el "centro de esta afectividad" (Ibid.). Así pues, desde un ángulo que conjuga semántica, ontología y fenomenología, el corazón posee dos significados; de los cuales no estamos eximidos de distinguir:

1) El corazón: raíz de la afectividad. Así como la potencia intelectiva es la raíz de todos los conocimientos, el corazón lo es con respecto a los estados afectivos particulares, tanto simples como profundos. Por consiguiente, "el corazón es el órgano de toda la afectividad: todos los deseos y anhelos, todo 'conmoverse', todos los tipos de felicidad y dolor están enraizados en el corazón en un sentido más amplio" (Ibid.). Aquí todavía estamos fenomenológicamente en una afectividad declinada de diversas maneras en sus múltiples afecciones, toda vez que nos apercibimos de la incompletud de estos y de la necesidad de cruzar el umbral hacia su raíz.

2) El corazón: centro de la afectividad. En comparación con el significado incompleto anterior, en el cual el corazón es entendido con respecto a otros estados afectivos, aquí vamos al origen mismo en su focalidad principal; siendo inclusive centro del mismo intelecto como Yo profundo del yo personal. Es mejor hablar de centro que de origen porque, según el criterio de Von Hildebrand, el yo vive la afectividad como un todo en diferentes grados y no como una cosa (origen afectivo) que causa otras (afectos). Así pues, el alemán se place en hablar de una única esfera afectiva que se puede experimentar tanto de manera periférica-marginal como nuclear. En comparación con la marginalidad del primer significado, en este llegamos "al verdadero núcleo de esta esfera" (Ibid.). La fenomenología hildebrandiana se propone alcanzar el "punto focal de la esfera afectiva, (...) que resulta afectado de modo más crucial", dando cuenta del "verdadero centro de gravedad de toda la afectividad" (Ibid.). Nos emplazamos, por ello, ante una fenomenología que ambiciona gravitar, con toda la connotación física y atrayente de este verbo, en el epicentro de 
la afectividad del yo. La fenomenología de la afectividad hildebrandiana ostenta un talante positivo, no solamente para con los sentimientos sino también hacia las pasiones; siempre y cuando no rebajen a simple animalidad la espiritualidad humana.

\subsection{Fenomenología de Los SENTIMIENTOS}

En esta tesitura no-dualista, la única "esfera afectiva" abarca fenomenológicamente diferentes experiencias sentimentales que se diversifican "en estructura, cualidad y rango" (Von Hildebrand, 2009, p. 60), tanto en los estados no-espirituales como en los espirituales. Nos resultará, a la sazón, francamente elemental no confundir ni dualizar la esfera afectiva, como todo experimentado, con los sentimientos que son una parte de la misma. Procedamos, pues, a tratar la fenomenología del alemán en sus diversos tipos de experiencias afectivas sentimentales:

1) Sentimientos físicos. En la fenomenología hildebrandiana, la "primera diferencia fundamental" (Ibid.) es la consistente en los sentimientos físicos. Son los sentimientos que el yo vive con más acopio en el cuerpo, siendo "evidentemente, experiencias conscientes, y están separados por un insalvable abismo de los procesos fisiológicos aunque guardan con ellos la más estrecha relación causal" (Ibid.). Otra forma de caracterizar estos sentimientos es denominarlos "experiencias corporales" en tanto que "sucesos que tienen lugar dentro del cuerpo" (Von Hildebrand, 2009, p. 61). En el dolor de cabeza, por ejemplo y en comparación con la tristeza, hay una gran diferencia ya que el primero se vincula con una parte del cuerpo y gravita sobre esa parte; mientras que la tristeza puede ser provocada por un deseo o frustración y no por la afección directa de una parte corporal.

El alemán califica, además, a los sentimientos físicos como "instintos corporales", siendo los únicos "que tienen una relación fenomenológica con el cuerpo" (Ibid.), constituyendo su voz en nuestra conciencia. Estos sentimientos son los que vivimos con más agudeza, consciencia y proximidad existencial, ya que, en oposición a los estímulos animales, "en el hombre no son ciertamente experiencias espirituales, pero son sin lugar a dudas experiencias personales" (Von Hildebrand, 2009, p. 62). Por tanto, estamos conminados a admitir que hay "un puente infranqueable" entre animales y seres humanos puesto que, en el hombre, "todo es radicalmente distinto al estar insertado en el mundo misteriosamente profundo de la persona y al ser vivido y experimentado por un yo" (Ibid.). En In defense of purity (Von Hildebrand 1931), publicado originalmente a comienzos de la tercera década del siglo pasado, el alemán llevaba esto al plano conyugal, al no poder aislar, son pena de deshumanizarnos, el sentimiento corporal de la intentio unionis y el marco estable de amor del desposorio. Y es que, para que el sentimiento reciba su cualificación 
fenomenológica humana, ha de estar en relación con toda la persona y su condición relacional.

2) Sentimientos psíquicos. Aquí tenemos los llamados "sentimientos nocorpóreos" (Von Hildebrand, 2009, p. 64), los cuales no gozan de la misma cualidad ni fenomenológica ni ontológica (no es lo mismo el buen humor que la tristeza, por poner un caso). Hemos de decir, además, que "estos estados psíquicos no tienen por qué estar causados por procesos corporales" (Ibid.). De hecho, los sentimientos psíquicos "no se presentan <específicamente> como la 'voz' de nuestro cuerpo ni son estados de nuestro cuerpo" (Ibid.). Esto se explica en que podemos sentir espiritualmente una alegría generalizada, aunque, tal vez por una desgracia fortuita, sintamos dolor en alguna parte de nuestro cuerpo. Sin duda, hay sentimientos físicos que acompañan y repercuten, tanto positiva como negativamente, en nuestros sentimientos psíquicos. Pero no son iguales. Lo más sano y deseable sería indudablemente que los sentimientos psíquicos y lo físicos se armonizasen en la persona total; lo cual no suprime la "diferencia básica" entre unos y otros. Esta diferencia, y conviene que lo señalemos, la descubrimos en el orden fenomenológico y no desde el modelo substancialista y dualista; al estilo de la doble substancia cartesiana.

3) Diferencia entre estados psíquicos y respuestas espirituales afectivas. Aunque léxicamente nos parezcan homogéneas, no debemos confundirlos ya que se basan en la diferencia entre intencionalidad y motivación. A tenor de Von Hildebrand, los estados psíquicos son siempre intencionales, no habiendo ningún acto consciente completamente ajeno a su marca": "La intencionalidad, en este sentido, es precisamente una marca esencial de la espiritualidad" (Von Hildebrand, 2009, p. 66). En cambio, en las respuestas espirituales afectivas, además de la ineludible intencionalidad, lo que tienen es una motivación, es decir, una "relación consciente y significativa con un objetivo" (Ibid.). Igualmente, hay una diferencia entre la causación que un estado físico comporta en uno psíquico (o uno psíquico en otro estado psíquico) y esta misma motivación que apuntamos. Expliquémonos con el recurso de ejemplos. A veces, la ingesta excesiva de bebidas espirituosas causa, directa y seguidamente, un buen humor asociado a la embriaguez, mientras que la presencia de la persona amada motiva la alegría como respuesta espiritual afectiva en todo

\footnotetext{
${ }^{2}$ Los únicos que pueden tildarse de no-intencionales son los sentimientos informales y transitorios, tales como los "humores irracionales que no son la resonancia legítima de una experiencia espiritual y que por lo tanto no están justificados ni son significativos, sino que son el efecto, o bien de causas corporales, o de experiencias que no justifican de ninguna manera estos estados de ánimo" (Von Hildebrand, 2009, p. 69). Estos humores son desproporcionados afectiva y racionalmente, por lo que su ilegitimidad viene dada porque se quiere justificar la experiencia espiritual en base a la inmanencia de los humores. En otras palabras, diríamos que los estados de humor no hacen la realidad, sino que es más bien lo contrario. Los "sentimientos no-intencionales como el mal humor, la depresión o la irritación, tienen el carácter de un estado psíquico y que pueden ser causados por procesos corporales o por causas físicas" (Von Hildebrand, 2009, pp. 86-87) no pueden ser la medida absoluta de la realidad ni de la verdad de la vida personal.
} 
caso superior: "presupone el conocimiento de un objeto y de su importancia" (Von Hildebrand, 2009, p. 67).

El alemán no quiere postular, de nuevo, la fractura cartesiana del alma y el cuerpo, ya que dichas respuestas espirituales repercuten también en nuestra salud física como en un todo. Se trata, pues, de sentimientos espiritualmente motivados que nos diferencian de los animales y nos recuerdan que no somos seres rebajadamente estimúlicos; alejándonos, por ende, de toda suerte de sentimentalismo. Comprobamos, pues, que estamos facultados para "emancipar nuestro intelecto y nuestra voluntad de la esclavitud de estos humores irracionales" (Von Hildebrand, 2009, p. 69). Mas, la emancipación a la que estamos autorizados naturalmente no es total, en contra del sueño moderno, sino relativa al cuerpo; so pena de resucitar el viejo espíritu cartesiano.

Antes de centrarnos en el próximo punto, hemos de indicar al lector que, ora en los sentimientos ora en las pasiones de la sola esfera afectiva, descubrimos dos formas de dependencia afectiva para con nuestro cuerpo. Estudiemos, a continuación, cómo dependemos del mismo, especialmente en las variadas maneras corporales que tienen - fenomenológicamente- un calado vivencial propio y distinto:

a) Dependencia consciente. Esta dependencia se vincula con la misma facultad de emanciparnos de manera relativa del cuerpo: se trata de la actitud afectivamente consciente ante la influencia corporal. Aquí encontramos dos tipos de dependencia consciente. En el primer tipo, hay quienes "se abaten completamente ante el dolor corporal o se ensimisman ante las molestias físicas o las incomodidades" (Von Hildebrand, 2009, 70), es decir, tenemos abatimiento y ensimismamiento como tonalidades dependientes. Por otra parte, y en el segundo tipo, hay quienes "muestran una gran independencia con respecto de su cuerpo", es decir, se mantienen en libertad y goce espiritual relativo, "aunque su cuerpo esté sometido a dolores" (Ibid.); siempre que estos no sean extremos o violentos.

b) Dependencia inconsciente. Aquí nos encontramos con "una dependencia de estados de ánimo psíquicos que en realidad están causados por nuestro cuerpo (...)" (Ibid.). De hecho, no es consciente, a primera vista, la influencia corporal en nuestro estado anímico. Como ejemplo de esto, tendríamos la falta de concentración o el irritamiento, a causa de procesos fisiológicos de los que no somos conscientes.

El error que solemos cometer aquí es que estos estados "no tienen bases racionales y se perciben erróneamente como una situación real de nuestra alma" (Von Hildebrand, 2009, p. 71). Se trata de la confusión anti-fenomenológica del cuerpo con la conciencia. A diferencia de la dependencia afectiva anterior, en la cual ciertamente podíamos tomar conciencia tanto para dejarnos influir como para, en el mejor de los casos, enseñorearnos de nuestros sentimientos, aquí los 
procesos netamente fisiológicos, "son tomados como si fueran psíquicos y como si fueran estados de nuestra alma" (Ibid.).

De este modo y al no ver que nuestra facultad consciente, incluso para sobreponernos a estos estados, sigue activada, de modo que nos "hacemos esclavos de nuestros cuerpos en un grado mayor que en el caso precedente" (Ibid.). Esto ocurre, por sacar un caso a la palestra, en la depresión; en la cual se revuelve un estado corporal aminorado con nuestro acceso natural consciente al mundo. Y es que, como nos señala Von Hildebrand, si tomáramos distancia consciente de esta revulsión podríamos, tal vez y con la debida ayuda médica, hallar vías para superar el estado depresivo.

\subsection{FenOMENOLOGÍA Y TIPOLOGÍA DE LAS PASIONES}

Pero en este trabajo no solamente hablamos de sentimientos. Von Hildebrand, tras ir acreditando en su fenomenología el gran cromatismo afectivo de la persona humana, nos exhorta a no generalizar el término de origen latino "pasiones" (passiones) a toda nuestra afectividad. Históricamente esto se ha hecho en nombre de un vilipendio -incluso en las teologías de la perfección cristiana- de todo lo que no fuera racional, guiándose por la instrumentalización del cuerpo. El alemán, en lo que a sí respecta, cree que las pasiones son una parte del todo afectivo humano y no pueden desacreditarse.

Más concretamente y en un significado primario, debemos entender por ellas los sentimientos "que alcanzan un alto grado de intensidad" y que "tienden a silenciar la razón y a dominar a la voluntad libre" (Von Hildebrand, 2009, p. 73). Las pasiones, entre las cuales podríamos incluir la ira, ofuscan la inteligencia y debilitan la libertad; atenuando en ocasiones la responsabilidad moral. Por no solo tenemos la ira. Hemos de acometer, por añadidura y como hacía el alemán en Transformation in Christ (Von Hildebrant 2001), una "distinción importante y hasta fundamental" -como en las dos "locuras" de la filosofía platónica- entre dos modos de éxtasis o de "estar fuera de sí" (Von Hildebrand, 2009, p. 73) que son radicalmente opuestos como acicate para una teología ejercida autrement:

a) Éxtasis falso o inferior. Es lo que el autor retrata como "pérdida de control ilegítima (...que) se caracteriza por la irracionalidad" (ibid.). Con manifiesta reciedumbre, el alemán descalifica este éxtasis como "brutal dinamismo", que no es espiritual, ya que "nuestro centro espiritual libre resulta superado y la persona arrojada en un brutal dinamismo biológico" (Von Hildebrand, 2009, p. 74). Un triste caso de esto sería el del drogodependiente que sale de sí no solo a causa de los efectos de las substancias alucinógenas que se aplica sino en razón de su ausencia con el síndrome de abstinencia. 
Por lo demás y en este nivel extático inferior, el alemán incluye la noción nietzscheana de lo "dionisíaco" (Von Hildebrand, 2009, p. 76), en la que el ser humano es hecho títere de la vida en su forma más ciega; como puede ser un deseo sexual descontrolado que ni tan siquiera respeta la integridad y libertad del otro. Hemos de contar, además, entre las formas de este desquiciado "fuera de sí" a la ambición, el resentimiento y la avaricia; en las cuales el centro espiritual humano es "absorbido por la búsqueda eficiente y 'razonable' de los fines propuestos por la pasión" (Von Hildebrand, 2009, p. 74). Por desgracia y en una dependencia casi total para con el cuerpo, el "fin último y verdadero de la razón que consiste en reconocer la verdad y en determinar lo que debemos hacer, lo que es moralmente recto, se frustra por el dominio de la pasión" (Ibid.). Contrariamente a esto, la racionalidad y la moralidad se conservarán en el próximo tipo de éxtasis.

b) Éxtasis elevado. En este caso opuesto al anterior, gozamos de la experiencia "de ser poseídos <sic.> por algo más grande que nosotros", en lo que el filósofo llama una "extraordinaria elevación" (Ibid.). Perdiendo la tierra firme de las experiencias corrientes y del dominio plenipotenciario de la razón, en el verdadero éxtasis constamos de "una toma de conciencia intuitiva que, en vez de ser irracional, tiene más bien un carácter suprarracional y luminoso" (Von Hildebrand, 2009, 75).

Este éxtasis no es un estado meramente sobrenatural, sino que es propio del ser humano en sus vivencias más extraordinarias, tanto en lo estético como en lo religioso (no solamente en lo religioso en sentido positivo o revelado sino de una vinculación natural afectiva para con una totalidad misteriosa). La prueba de que el éxtasis es fuertemente humano es que Von Hildebrand lo incluye entre las pasiones, algo muy rompedor en comparación con ideologías pseudocristianas tendentes a espiritualizar en demasía al ser humano. El éxtasis es un estado pasional insigne (quizás enaltecido por incitaciones instrumentalistas desde cierto quehacer teológico), pero es pasión a fin de cuentas. Es más, el éxtasis es el culmen de la pasión. De este modo y aunque así se pueda interpretar, no es que Von Hildebrand anule la razón, como en el falso éxtasis, para biologizarnos en un mal sentido; sino que se le concede una perspectiva nueva a diferencia de sus solas herramientas inductivodeductivas.

En vez que subyugar a la razón con violencia, en el éxtasis genuino más bien se "reclama la sanción de nuestro centro libre" (Von Hildebrand, 2009, p. 75), de tal suerte que la podemos fenomenologizar como la antítesis de toda esclavización o avasallamiento de la condición espiritual humana. Antes al contrario, este éxtasis, transido de "claridad intuitiva", puede ser comprendido como "liberación de las cadenas que nos mantienen en la tierra" (Ibid.). Más que la ataraxia y la apatheia helénicas, que no acaban de integrar las diferentes dimensiones personales, este éxtasis engrandece a la persona; de tal modo que se aproxima más a la sere- 
nitas animae de los estados de beatitud en una especie de "confiado abandono" (Von Hildebrand, 2009, p. 76) según la teología clásica; ya que no se destrona, incluso en este estado elevado, a la inteligencia y la voluntad.

Para resumir su fenomenología de las pasiones, Von Hildebrand las recapitula, en función de su no-inclusión en el seno de la racionalidad, en una tipología cuádruple de la experiencia afectiva, gozando cada tipo de su estatus ontológico propio. En el primer caso hallamos las pasiones stricto sensu (ambición, deseo de poder, codicia, etc.), que tienen un "carácter oscuro y antirracional" (Von Hildebrand, 2009, p. 80). En el segundo tipo descubrimos las pasiones explosivas (principalmente la ira) que no vienen espiritualmente motivadas por alguna causa justa, sino que es "la ira qua ira que posee un carácter explosivo, incontrolado e impredecible" (Ibid.). Para continuar con la tipología, nos situamos además ante las pasiones impulsivas que "esclavizan a la persona" (Von Hildebrand, 2009, 81); como en el caso de las adicciones al alcohol o a las drogas.

Finalmente, hemos de mencionar las pasiones de respuesta, que son "respuestas afectivas que a pesar de tratarse de respuestas al valor, pueden escapar a nuestro control" (Ibid.); como es el caso del amor pasional descontrolado en los amantes y el cual no exhibe, a causa de su arrebato, la misma altura de las respuestas espirituales afectivas que ya hemos estudiado.

\section{EL ETHOS ESPECÍFICO DE LA AFECTIVIDAD}

Von Hildebrand da un paso hacia delante en su fenomenología de la afectividad ya que, si acabamos de abordarla sistemáticamente en su amplia gama sentimental y pasional, ahora inquiere un "sentido más estricto" referido a un "solo tipo particular de sentimientos"; tratándose en consecuencia de un "ethos específico" diferente de la "afectividad en sentido amplio" (Von Hildebrand, 2009, p. 89). El alemán opta por este término griego debido a que expresa convenientemente la costumbre y el aparecer concentrado de la afectividad toda. Aquí podremos hallar elementos para seguir provocando a la teología hors jeu con respecto a la fenomenología.

\subsection{LA AFECTIVIDAD TIERNA}

El primer ethos específico de la afectividad que Von Hildebrand resalta es la ternura como reacción a las corrientes anti-afectivas modernas y como defensa de la afectividad de la persona, esto es, una que no reduzca o simplifique ninguna de sus dimensiones. En efecto, después de la Gran Guerra "y como consecuencia del ethos del siglo XIX, surgió una fuerte tendencia anti-afectiva" (Von Hildebrand, 2009, p. 91). Dicha propensión se desarrolló fundamentalmente en el arte, pero también, 
se lamenta el alemán, "en la esfera de las manifestaciones religiosas” (ibid.). La teología que piensa estas manifestaciones ha de ser provocada.

Aunque el sentimentalismo religioso era algo que urgía corregir, "no se buscó la solución en una afectividad genuina sino en el rechazo de todo tipo de afectividad" (Von Hildebrand, 2009, Ibid). Por desgracia, toda la afectividad, sin los distingos fenomenológicos que ya hemos efectuado en el punto 3, era recriminada como un "subjetivismo trivial" en contraposición a la "sólida sobriedad (...) del espíritu de objetividad" (Von Hildebrand, 2009, Ibid). Y es que nos indica Von Hildebrand que la ideología imperante en la época contemporánea es la de un funcionalismo, o como se ha estilado en el arte, el de una Neue Sachlichkeit o Nueva Objetividad. En efecto, todo rasgo de ternura afectiva es motivo de un desmedido demérito y considerable preterición, bajo las consignas de una "racionalidad fría y un pragmatismo utilitarista" (Von Hildebrand, 2009, p. 93).

Como invectiva contra esto, el alemán incluye a la ternura en el "carácter específicamente humano y personal" de la efectividad y ataca con ingenio que, si por un lado se repulsa la ternura desde la racionalidad moderna, no nos damos cuenta que el sentimentalismo que se busca superar es todavía más impotente en su propia solución funcional-utilitaria, ya que no se denuncian otros sentimientos, tremendamente afectivos, como "la ambición, el deseo de poder, la codicia o la lascivia" (Von Hildebrand, 2009, Ibid). En la Modernidad no se denuncia esta afectividad desordenada o "afectividad enérgica" (Von Hildebrand, 2009, p. 102) sino que, antes al contrario, está bien vista en contra del corazón o afectividad tierna. Uno de los sentimientos propios de la iluminada y fría Modernidad (jsentimiento afectivo también!) es el de un "egocentrismo mezquino" (Von Hildebrand, 2009, 97), del hombre que se cree el centro del universo, pero no somete su superioridad a ninguna auto u altero-crítica. ¿No se podría beneficiar de esta crítica a la Modernidad la teología moral actual?

La Modernidad, que quiere emanciparse del sentimentalismo y al no hacer una fenomenología de la afectividad, se hace de nuevo rea del mismo en una efectividad enérgica incriticada: "condenan a toda la esfera de la afectividad tierna por ser subjetiva, blanda y ridícula" (Von Hildebrand, 2009, p. 93). Así pues, el filósofo aprovecha la ocasión para fustigar un vitalismo biologicista antipersonal, cuyos "seguidores de este ídolo consideran que las tendencias biológicas como los instintos son algo más ‘orgánico’ y genuino que cualquier acto espiritual consciente” (Von Hildebrand, 2009, p. 98). La Modernidad -de la cual la teología no ha sido ajena en su desarrollo- es el ejemplo de la defensa de una afectividad total que, al ser imposible de derrotar, es traicionada, acallada y falsificada en un cierto tipo de "anti-personalismo que encierra la tendencia anti-afectiva (...) en una antipatía contra la 'conciencia' (consciousness)" (Von Hildebrand, 2009, Ibid). La noción 
moderna de conciencia no es entendida por el alemán como introversión solipsista y subjetivista ${ }^{3}$ sino como "una experiencia más plena y despierta" (Von Hildebrand, 2009, pp. 98-99), una conciencia cordial del mismo mundo y la naturaleza. Tener corazón es experimentar afectiva y conscientemente la realidad sin cortapisas ni reduccionismos ideológicos en la integridad de la persona humana.

$\mathrm{Y}$ es que, siendo honestos en nuestro análisis fenomenológico, "cualquier tendencia antiafectiva, por lo tanto, es en realidad un subjetivismo patente porque su respuesta al mundo es incorrecta" (Von Hildebrand, 2009, p. 102), queriendo negar el corazón del que nadie se puede eximir naturalmente. Por ello, el alemán distingue entre la "efectividad meramente temperamental y sin corazón" (Von Hildebrand, 2009, pp. 93-94) de la afectividad tierna del corazón, la cual, siendo hondamente personalistas, nos viene descrita de este modo:

La afectividad tierna se manifiesta en el amor en todas sus formas: amor paternal y filial, amistad, amor fraterno, conyugal y amor del prójimo. Se muestra al 'conmoverse', en el entusiasmo en la tristeza profunda y auténtica, en la gratitud, en las lágrimas de grata alegría o en la contrición. Es el tipo de afectividad que incluye la capacidad para una noble rendición y en la que está implicado el corazón (Von Hildebrand, 2009, Ibid).

\subsection{Hipertrofias Y ATROFIAS AFECTIVAS}

En continuidad con su crítica de la Modernidad pero también del pietismo religioso de propagados sectores teológicos, el filósofo alemán nos previene de lo que él caracteriza como "hipertrofia del corazón” (Von Hildebrand, 2009, p. 111). Esto es una forma aseadamente metafórica que conduce con vehemencia a "un uso excesivo de la afectividad que en realidad es un uso incorrecto" (ibid.); al pretender desordenar y desajustar la experiencia personal que nos cualifica como humanos:

El desorden se produce porque el corazón, en vez de cooperar con el intelecto y la voluntad, o bien intenta realizar lo que solo el intelecto puede llevar a cabo correctamente, o bien se niega a conceder a la voluntad su misión específica (ibid.).

Como se puede colegir a partir de la anterior cita, el intelectualismo y el voluntarismo son desórdenes afectivos en la fenomenología hildebrandiana. Una persona alienada en un puro hacer, en una ciega tendencia a objetos y proyectos, vive su afectividad de manera palmariamente cercenada. La afectividad, además de la voluntad, implica amar, el recto desinterés y la quietud ante experiencias enriquecedoras como la contemplación o la meditación. Es por ello que actuar sin pensar y ni reflexionar no

\footnotetext{
${ }^{3}$ Para Von Hildebrand (2009), pp-99-100, no debería haber riñas entre afectividad y objetividad ya que "la afectividad tierna implica (...) que una actitud se adecúa a la verdadera naturaleza, tema y valor del objeto al que se refiere (...) responde al bien que es la base y la fuente de su experiencia afectiva".
} 
es menos deletéreo para la persona. La misma crítica nos vale para el intelectualista, que, por ejemplo, se priva de las potencialidades de su capacidad de acción y de empatizar sentimentalmente con los otros. Voluntarismos e intelectualismos, tanto en filosofía como en teología, son hipertrofias afectivas porque ponemos en ellos, no el corazón en su toda su hondura con la esfera afectiva restante, sino en los fragmentos cognoscitivos, volitivos o amativos. En efecto, la hipertrofia del corazón consiste en amar con y en uno de estos fragmentos, con el apuro que esto comporta de no amarse a sí mismo ni a los demás completamente:

(...) si la hipertrofia del corazón constituye un peligro, lo mismo sucede con una hipertrofia del intelecto y de la voluntad por lo que la cooperación del intelecto, de la voluntad y del corazón es la de la mayor importancia para todos y cada uno de ellos (Von Hildebrand, 2009, p. 112).

La afectividad, en términos hildebrandianos, es la esfera armónica de las dimensiones intelectivas, volitivas y amativas. Sin embargo, hipertrofia del corazón no significa demasiada intensidad en amar: una afectividad armónica como la que defiende el filósofo alemán, de ser intensa en el acto de amar integral, es muy beneficiosa para la persona; si bien el problema reside en el desorden y la intensificación ilusoria de alguna dimensión afectiva sobre otra: "Cuanto más grande sea la capacidad afectiva del hombre, mejor" (Von Hildebrand, 2009, p. 111).

Cuando esta grandeza afectiva se desmigaja, padecemos lo que el alemán bautiza como "atrofia afectiva" (Von Hildebrand, 2009, p. 113), que es la contraparte de las hipertrofias que hemos explicado. La hipertrofia afectiva es directamente proporcional a la atrofia afectiva y viceversa: la afectividad al ser una única esfera es la que se puede atrofiar, mientras que los afectos desordenados al tener una cierta especificidad se pueden hipertrofiar. Para el filósofo, "existen diversos tipos de hombres en los que la afectividad está mermada o frustrada", a los cuales incumbe un tipo de "afectividad mutilada" (Ibid.) en directa proporción con la hipertrofia de una dimensión de esta misma afectividad. En vez de estigmatizarlos desde la óptica moral, se destapan fenomenológicamente tres tipos de "eunucos afectivos" (Ibid.) a los que una teología más cordial no podría escudar:

1) En la hipertrofia del intelecto se atrofia la afectividad total ya que "es como un quedar enjaulado por un hechizo de la investigación" (Ibid.), repercutiendo negativamente en la acción y en las relaciones plenas de la persona con lo otro, los otros y lo Otro. Estos, en vez de contar en su cualidad personal irreductible, son instrumentalizados a favor de su consideración intelectual y una curiosidad muy lejana de una búsqueda sincera de la verdad.

2) En la hipertrofia de la "eficiencia pragmática", la persona piensa que "toda experiencia afectiva es superflua y constituye una pérdida de tiempo" (Von Hilde- 
brand, 2009, p. 115), llegando en el peor de los casos a la "mofa de cualquier gesto de compasión por la persona que sufre" (Ibid.). En este segundo tipo también se encuentra, tal y como nos remite el alemán a The true morality and its counterfeits (Von Hildebrand y Jourdain 1995), el "burócrata metafísico"; el cual es describible como un "funcionario "fosilizado" y para quien "solo cuentan las cosas que tienen realidad jurídica" (Von Hildebrand, 2009, p. 115). En este caso tal persona reduce la afectividad "a la satisfacción que siente al cumplir a la letra las prescripciones legales" (Ibid.).

No podemos sino relacionar esto con la caracterización que hizo Hannah Arendt en 1964 del nazi Adolf Eichmann como un hombre de aspecto corriente pero sometido a una idea del deber por el deber que le llevaron a cooperar burocráticamente en los más horrendos crímenes del holocausto. Se trataba de un caso de banalidad del mal, en el que, como escribía la filósofa de origen judío, el "perfecto burócrata" (perfekten burokraten) mantenía - al margen de la moral afectiva- que "cumplir las leyes no significaba únicamente obedecerlas, sino portarse como si uno fuera el autor de las leyes que obedece" (Arendt, 2007, p. 233). Se trataba, pues, de una moral kantiana llevada al paroxismo.

3) En la "hipertrofia de la voluntad", Von Hildebrand (2009), p. 116, nos advierte que "el empequeñecimiento de la esfera afectiva es generalmente algo deliberado". En este desorden afectivo, sea bajo el pretexto de la moral formal kantiana, una práctica religiosa basada exclusivamente en la repetición de los actos o quizá en la apatheia estoica, la "voluntad, a propósito, reduce toda la afectividad y silencia el corazón" (Ibid.).

\subsection{FALTA Y TIRANÍAS AFECTIVAS}

En nuestra experiencia fenomenológica no solamente nos encontramos con la atrofia afectiva en relación con alguna hipertrofia de afectos concretos, sino también con lo que engañosamente llamamos "falta de afectividad". Ni siquiera a esto da tregua la fenomenología hildebrandiana en su interés exhaustivo. De hecho, Von Hildebrand distingue entre "falta de corazón (heartlesness)" y "la impotencia afectiva" o "afectividad tullida" (Von Hildebrand, 2009, p. 116). La razón de esta diferenciación es que no puede haber la misma valoración moral en una o en otra.

Es, pues, moralmente peor la falta de corazón porque concierne al centro espiritual del hombre y, por tanto, a su libertad más profunda; mientras que la afectividad impotente no se debe a limitaciones no auto-impuestas sino provenidas de las carencias mismas de la persona en su desarrollo biológico, educativo y afectivo: "La falta de corazón se refiere a la mutilación de un centro en el alma del hombre" (Ibid.), como es el caso del "hombre despiadado o duro de corazón" 
(Ibid.). Notemos que el alemán no adopta con esta crítica la postura anti-afectiva contra la que él mismo se querella.

En otras palabras, diríamos que "el corazón acallado y helado del hombre al que le falta corazón" (Ibid.) no es sino apariencia de falta afectiva: mientras más exterioriza el hombre su falta de la misma, más demuestra su presencia: la de un corazón moralmente malvado en su silencio y que se ha empecinado en no amar. No es que sea incapaz de amar, sino que ha deliberado no hacerlo. Puesto que el yo más real y nuclear de la persona es el corazón tierno, la ausencia presuntamente total de ternura proviene de este mismo centro. Hay cuatro tipos de falta de corazón -o de discordialidad - a la que ninguna filosofía ni ninguna teología pueden permanecer sordas:

1) Egoísmo discordante. En el primer tipo de falta de corazón hallamos a las personas que solo buscan satisfacer su ego y son propensas a la inmoralidad. Son, por consiguiente, aquellas "personas a las que solo importa una cosa: la gratificación de su orgullo y de su concupiscencia" (Von Hildebrand, 2009, p. 120).

2) Cinismo discordante. "El segundo tipo de 'falta de corazón' puede deberse a ciertas pasiones como la ambición o la avaricia que sofocan el corazón del hombre o lo endurecen" (Von Hildebrand, 2009, p. 123). También se pueden contar en esto a las otras pasiones, "siempre que se realicen con cinismo" (Ibid.). En otras palabras, el cinismo es visto por el filósofo alemán como una manera de entumecer el corazón mediante la grosería y las acciones vituperables de humillar al otro para enaltecer el propio ego.

3) Esteticismo discordante. "Un tercer tipo de falta de corazón se puede encontrar en el esteta refinado" (Ibid.). Prosiguiendo las aclaraciones del alemán, a esta persona solo le interesa el carácter estético, el brillo superficial, y no el corazón de los demás. Recordemos la obsesión por la estética del partido nazi. El esteta puede llegar a la crueldad y a la total falta de empatía para lo que no se case con sus cánones de belleza.

4) Indolencia discordante. Esto se trata del "hombre amargado" (Von Hildebrand, 2009, p. 124), según leemos en el alemán. Podría acontecer que alguien aplaque su corazón "por un trauma mayor, por una herida en su corazón” (Ibid.). De este modo, este tipo de endurecimiento tiene un "carácter trágico" (Ibid.) a causa de una eventual traición relacional o, simple y llanamente, en razón de la impericia y poca habitualidad en experimentar el amor.

La fenomenología hildebrandiana es muy indicadora no solo para distinguir la presencia de la ausencia del corazón, sino al hacernos apreciar que la presumible falta total de corazón -incluso en el quehacer teológico- no deja de ser sino un 
corazón doliente o malogrado, que pretende finiquitarse infructuosamente: el corazón sigue latiendo incluso para cerrarse al amor. Por ello, la cara oculta de su falta es la tiranía del corazón. No solamente es tiránico el corazón que se niega a amar (porque deniega su más preciado don a sí y a los demás apoyándose en su poder de amar) sino el corazón como desordenadamente predominante con respecto a las dimensiones volitivas y afectivas de la esfera afectiva:

Este corazón tiránico aparece siempre que el corazón se niega a permitir que el intelecto decida lo que solo puede decidir el intelecto, o cuando se opone a que el centro libre y espiritual de la persona intervenga con un acto voluntario en el área reservada a la voluntad (Von Hildebrand, 2009, p. 127).

Sin duda que el corazón es el centro de la esfera afectiva, pero esto no equivale, de ninguna manera, a cordializar de manera asfixiante y despótica toda la persona en detrimento de su intelecto y voluntad. La persona es tan irreductible que ella puede y debe saber cómo amar y cómo pautar su amor mediante las acciones. Cuando reducimos la persona a un corazón exaltado y fanático la privamos de la posibilidad de amar más y mejor porque, para ser veraces, el ejercicio simultáneo del intelecto y la voluntad le sirven para autentificar y poner en marcha su cordialidad no solo con mayores cotas de éxito sino de moralidad. En imágenes parejas a las de Von Hildebrand, podríamos afirmar que un amor sin cabeza y sin pies es un amor que no se adecúa al bien integral de la persona, un amor expuesto a ser un tirano contra las razones esgrimidas y la tendencia a los valores morales.

Por ello, "el corazón tiránico se manifiesta también en algunas debilidades que resultan de una benevolencia desordenada" (Von Hildebrand, 2009, p. 128), sobre todo en "personas incapaces de rechazar cualquier petición a menos que se trate de algo estrictamente pecaminoso" (Ibid.). Así y en una suerte de debilidad e injusticia, estas personas “demasiado buenas” (según la denominación hildebrandiana) parecen ignorar "que el verdadero amor nos obliga a pensar en el bien objetivo de nuestro prójimo y no a satisfacer todos sus deseos" (Ibid.). Muchas veces y en esto nos es muy iluminador Von Hildebrand, “un 'no' puede ser una manifestación mucho más verdadera de amor que un 'sí'”, (Ibid.).

Otra tiranía que describe magistralmente el alemán es "la mediocridad del corazón", la cual consistiría en un "egocentrismo mezquino que se toma muy en serio cualquier nimiedad que concierne al propio yo" (Von Hildebrand, 2009, p. 129) y el cual es proclive principalmente en personas demasiado susceptibles afectivamente que se niegan o no saben medir objetivamente el mal que supuestamente padecen de parte de otras personas. Muchas veces y sin confundir esto con las personas verdaderamente sencillas (la sencillez, si es espiritualmente integrada, nos sería válida con vistas a una afectividad plena), los mediocres solo pretenden "principalmente la 
satisfacción de su propia vanidad y malgastar su tiempo preocupándose de ofensas imaginarias" (Ibid.).

Queremos consumar nuestro artículo con un caso a no desatender. Finalmente, y sin la irascibilidad del ejemplo anterior, la última tiranía del corazón (también mediocre pero apocada) es la de "un desordenado deseo de ser amado" (Von Hildebrand, 2009, p. 62) en personas que siempre se sienten maltratadas, ignoradas o arrinconadas ante escarnios tanto reales como imaginarios y al no tener un claro dominio y autoconciencia de su persona. Estas personas se infravaloran considerablemente y, en vez de encarar las afrentas, se encierran en sí mismas y se auto-compadecen demostrando con ello su falta de madurez afectiva. La teología no ha de acentuar el ser amado de manera absoluta sin pensar antes y auspiciar un amor maduro. Para consumar este artículo, la rica afectividad humana, como colofón, ha quedado prolijamente fenomenologizada gracias a Von Hildebrand pudiendo así provocar cordialmente a la teología.

\section{BIBLIOGRAFÍA}

Arendt, H. 2007. Eichmann in Jerusalem. Ein Bericht von der Banalität des Bösen. München: Piper. Burgos, J.M. 2009. Presentación. En D. Von Hildebrand, El corazón. Un análisis de la afectividad humana y divina, pp. 5-30. Madrid: Ediciones Palabra.

Culleton, A. 2010. "Tres aportes al concepto de persona: Boecio (substancia), Ricardo de San Víctor (existencia) y Escoto (incomunicabilidad)". Revista Española de Filosofía Medieval $\mathrm{N}^{\mathrm{o}} 17$, pp. 59-71.

Von Hildebrand, D. 1931. In defense of purity: an analysis of the Catholic ideals of purity and virginity. New York: Longmans, Green \& Co.

Von Hildebrand, D. 1953. Christian Ethics. New York: David McKay Co.

Von Hildebrand, D. 1973. What is Philosophy? Chicago: Franciscan Herald.

Von Hildebrand, D. 1977. The heart: an analysis of human and divine affectivity. Chicago: Franciscan Herald.

Von Hildebrand, D. 2001. Transformation in Christ. On the Christian attitude. San Francisco: Ignatius Press.

Von Hildebrand, D. 2009. El corazón. Un análisis de la afectividad humana y divina. Trad. J.M. Burgos. Madrid: Ediciones Palabra.

Von Hildebrand, D. 2014. My battle against Hitler. Faith, truth and defiance in the shadow of the Third Reich. New York: Penguin.

Von Hildebrand, D y Jourdain, A.M. 1955. True morality and its counterfeits. New York: David McKay Co. 